\title{
Determining the Components of the Complement of a Digital (n-1)-manifold in $\mathbb{Z}^{n}$
}

\author{
R. Ayala ${ }^{1}$, E. Dominguez ${ }^{2}$, A.R. Francés ${ }^{2}$, A. Quintero ${ }^{1}$ \\ 1 Dpt. de Algebra, Computación, Geometría y Topología. Facultad de Matemáticas. \\ Universidad de Sevilla. Apto. 1160. E-41080 - Sevilla. Spain. \\ e-mail: almarecica.es \\ 2 Dpt. de Informática e Ingeniería de Sistemas. Facultad de Ciencias. \\ Universidad de Zaragoza. E-50009 - Zaragoza. Spain. \\ e-mail: cciapposta.unizar.es
}

\begin{abstract}
The goal of this paper is to determine the components of the complement of digital manifolds in the standard cubical decomposition of Euclidean spaces for arbitrary dimensions. Our main result generalizes the Morgenthaler-Rosenfeld's one for $(26,6)$-surfaces in $\mathbb{Z}^{3}$ [9]. The proof of this generalization is based on a new approach to digital topology sketched in [5] and developed in [2].
\end{abstract}

Keywords: Digital space, digital $n$-manifold, digital and topological index, Jordan-Brouwer Separation Theorem.

\section{Introduction}

Morgenthaler-Rosenfeld [9] define a digital surface $S$ as a subset of points in $\mathbb{Z}^{3}$ verifying certain local properties, and then they prove a digital version of the Jordan-Brouwer Separation Theorem. To prove this result, they define when a digital half-line intersecting the surface at a point crosses the surface at this point. The points $p$ in the complement $\mathbb{Z}^{3}-S$ of the surface $S$ are arranged into two classes according to the number (even or odd) in which a half-line emanating from $p$ and parallel to one of the coordinate axes crosses $S$. Finally, these classes are proved to be the connected components in which $S$ separates its complement $\mathbb{Z}^{3}-S$.

On the other hand, in [2] a definition of digital $n$-manifold, which generalizes the Morgenthaler-Rosenfeld (26,6)-surfaces (see [1]), is given and the Digital Jordan-Brouwer Theorem is also generalized to arbitrary dimensions. The proof makes use of an architecture, sketched in [5] and developed in [2], consisting of several levels which provide a link between digital spaces and Euclidean spaces. By using this architecture, the hypotheses of the quoted digital theorem are translated to the hypotheses of the well-known Jordan-Brouwer Theorem in topology, and then the thesis of the latter is translated back again into the thesis of the former. In this way, the proof is based on suitable translations of knowledge through the different levels of the architecture, and a new proof for the Digital Jordan-Brouwer Theorem, independent of that for the continuous result, is not needed. 
However, this proof only assures that a digital $(n-1)$-manifold $M$ in $\mathbb{Z}^{n}$ separates its complement $\mathbb{Z}^{n}-M$ into two components, but does not provide a method for determining in which one a point lies. Our main goal is to give such a method by characterizing those points which belong to a given component of $\mathbb{Z}^{n}-M$.

In terms of the usual language of adjacency pairs of Rosenfeld and other authors, we can say that a digital $(n-1)$-manifold in $\mathbb{Z}^{n}$ is a $\left(3^{n}-1,2 n\right)$ hypersurface; that is, one uses the $\left(3^{n}-1\right)$-adjacency for the hypersurface and the $2 n$-adjacency for its complement. Then, as in the continuous case, our main result (Theorem 4) characterizes the $2 n$-components of the complement $\mathbb{Z}^{n}-M$ of a hypersurface $M$ in terms of the notion of (digital) index, $i_{\text {dig }}(x ; M)$, of a point $x \in \mathbb{Z}^{n}-M$. Namely, $x$ belongs to the finite $2 n$-component of the complement of $M$ if and only if $i_{d i g}(x ; M)$ is odd. This method generalizes to arbitrary dimensions that from Morgenthaler-Rosenfeld [9].

To accomplish our objective, we begin in Section 2 by reviewing some definitions and results from [2]. Section 3 is aimed to state precisely our main result exclusively in digital terms. The proof of this result needs of some other results from polyhedral topology which are introduced in Section 4, and then translated into the digital level in Section 5. In Section 6, we prove that our method is actually a generalization of Morgenthaler-Rosenfeld's one, and give a simplified algorithm for the case $n=3$, which makes use of the characterization of the $(26,6)$-surfaces due to Kong-Roscoe [7].

\section{Previous notions}

In [2] a new approach to digital topology is introduced. In that approach a digital space is defined as an architecture made up of several levels which provides a bridge for transfering definitions, statements and proofs from continuous topology to digital topology.

In that approach the pixels on a computer screen are represented by the $n$-cells of a homogeneously $n$-dimensional locally finite polyhedral complex $K$. Namely, $K$ is a complex of convex cells (polytopes) such that each cell is face of a finite number (non-zero) of $n$-cells. If $\sigma$ is a face of $\gamma$ we shall write $\sigma<\gamma$. Given $\sigma \in K$, the boundary of the cell $\sigma$ is the set $\partial \sigma$ union of its proper faces, and the interior of $\sigma$ is the set $\stackrel{\circ}{\sigma}=\sigma-\partial \sigma$. If $|K|$ denotes the underlying polyhedron of $K$, a centroid-map is a map $c: K \rightarrow|K|$ such that $c(\sigma) \in \stackrel{\circ}{\sigma}$. We refer to [12] for all notions of polyhedral topology contained in this paper.

The polyhedral complex $K$ is called device level of the digital space.

Three other intermediate levels bridge the gap between the device level and the continuous one. All of these levels are determined by the device level $K$; so that, $K$ will also denote the whole digital space.

The nearest to the device level, called logical level of $K$, is an undirected graph $\mathcal{L}_{K}$ whose vertices are the centroids of $n$-cells in $K$, and two vertices are adjacent in $\mathcal{L}_{K}$ if their corresponding $n$-cells intersect. 


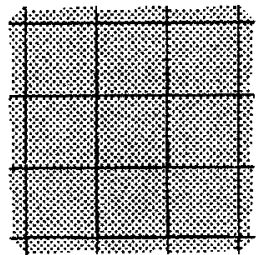

$R^{2}$

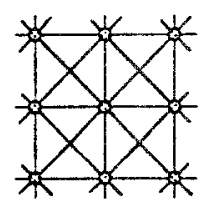

$\mathcal{L}_{R^{2}}$

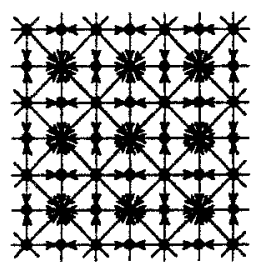

$\mathcal{C}_{R^{2}}$

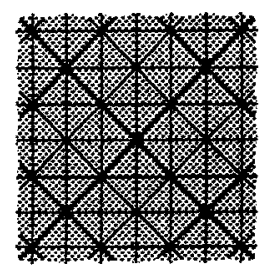

$\mathcal{A}_{R^{2}}$

Fig. 1. The device level $R^{2}$ and its logical, conceptnal and simplicial levels.

The digraph $\mathcal{C}_{K}$, called conceptual level of $K$, is defined as follows. Its vertices are those of $\mathcal{L}_{K}$ and, in addition, the centroids $c(\sigma)$ such that $\sigma$ is the intersection of two or more $n$-cells of $K$. The directed edges are pairs $(c(\tau), c(\sigma))$ with $\tau<\sigma$ (i.e., $\tau$ face of $\sigma$ ).

The simplicial analogue of $K$ is the order complex $\mathcal{A}_{K}$ associated to the graph $\mathcal{C}_{K}$. That is, $\left\langle x_{0}, x_{1}, \ldots, x_{m}\right\rangle$ is an $m$-simplex of $\mathcal{A}_{K}$ if $x_{0}, x_{1}, \ldots, x_{m}$ is a directed path in the digraph $\mathcal{C}_{K}$. This simplicial complex defines the simplicial level of the architecture and, finally, the continuous level is represented by the underlying polyhedron $\left|\mathcal{A}_{K}\right|$ of $\mathcal{A}_{K}$. This polyhedron is called the continuous analogue of $K$.

In this paper we will deal with the digital space $R^{n}$ defined by the standard cubical decomposition of the $n$-dimensional Euclidean space $\mathbb{R}^{n}$. That is, the device level of $R^{n}$ is determined by the collection of unit $n$-cubes in $\mathbb{R}^{n}$ whose edges are parallel to the coordinate axes and whose centers are in the set $\mathbb{Z}^{n} \subseteq \mathbb{R}^{n}$ of points with integer coordinates. The centroid-map we will consider associates to each cube $\sigma$ its center $c(\sigma)$. Hence, the logical level $\mathcal{L}_{R^{n}}$ is the graph of $\left(3^{n}-1\right)$-adjacencies whose vertex set is $\mathbb{Z}^{n}$ and two points $x, y \in \mathbb{Z}^{n}$ are adjacent if each one of their coordinates differs in at most 1 . The conceptual level is the digraph $\mathcal{C}_{R^{n}}$ which consists of the centers of all cells in $R^{n}$ as its vertices, and two of them are adjacent if one of their correspondig cells is a face of the other. Notice that $\mathcal{C}_{R^{n}}$ agrees with the $n$-dimensional Khalimsky's digital space (see [6] and [2]). Finally, the simplicial analogue $\mathcal{A}_{R^{n}}$ is a simplicial complex whose $m$-simplices are all the complete subgraphs with $m+1$ vertices of $\mathcal{C}_{R^{n}}$. Observe that $\mathcal{A}_{R^{n}}$ is simplicially isomorphic to the derived subdivision of $R^{n}$ induced by the centroids of its cells; and thus, $\mathcal{A}_{R^{n}}$ is a triagulation of the Euclidean space $\mathbb{R}^{n}$. In Figure 1 it is represented a portion of $R^{2}$, the standard cubical decomposition of the Euclidean plane, and its logical, conceptual and simplicial levels. Notice that the graph $\mathcal{L}_{R^{2}}$ is obtained by linking each point in $\mathbb{Z}^{2}$ with its 8-neighbours. In fact, when $n=2(n=3) \mathcal{L}_{R^{n}}$ is the graph of 8(26-) adjacencies used by Rosenfeld in his approach to digital topology (see [11] and [9]).

A digital object in a device level $K$ is a subset $O$ of the set of $n$-cells of $K$. Every digital object $O$ induces a digital space by regarding the subcomplex $K(O)=\{\sigma \in K \mid \sigma<\tau, \tau \in O\}$ as a new device model. Therefore, the logical 
and conceptual levels, and the simplicial and continuous analogues of $K(O)$ are naturally associated with $O$, and we will denote them $\mathcal{L}_{O}, \mathcal{C}_{O}, \mathcal{A}_{O}$ and $\left|\mathcal{A}_{O}\right|$, respectively. It is easy to verify that these levels of $K(O)$ are substructures of the corresponding ones of $K$. This justify to call $K(O)$ a digital subspace of $K$.

It is well-known that, to avoid certain paradoxes related to the Jordan Curve Theorem, in most of the graph-based models for digital topology must be defined two different notions of connection, one for the objects and the other for their complements. As the logical level of a digital space is also a graph, the following notions of connection are needed in this architecture. A subset $C \subseteq O$ is called a component of the digital object $O$ if $\mathcal{L}_{C}$ is a connected component of $\mathcal{L}_{O}$. On the other hand, a subset $D$ of $n$-cells is called a $c$-component of the complement of $O$ if the centroids of elements in $D$ are the vertices of a connected component of $\mathcal{C}_{K}-\mathcal{C}_{O}$. However, these notions can be established in terms of the usual notion of connectedness in the continuous analogue. Indeed, $C$ is a component of $O$ if and only if the continuous analogue $\left|\mathcal{A}_{C}\right|$ is a connected component of $\left|\mathcal{A}_{O}\right|$; and, there exists a 1-1 correspondence between the set of c-components of the complement of $O$ and the set of connected components of the topological space $\left|\mathcal{A}_{K}\right|-\left|\mathcal{A}_{O}\right|$. In fact, each $c$-component $D$ is determined by the $n$-cells of $K$ whose centroids are in a connected component of $\left|\mathcal{A}_{K}\right|-\left|\mathcal{A}_{O}\right|$ (see [2]).

With the above notation, a digital object $M$ will be called a digital $n$-dimensional manifold (without boundary) if $\left|\mathcal{A}_{M}\right|$ is a combinatorial $n$-manifold (without boundary); see Section 4 . Hereafter, manifold will mean combinatorial manifold. In this context a digital version of the Jordan-Brouwer Theorem can be proved by using the corresponding continuous result.

Theorem (Digital Jordan-Brouwer Theorem). Let $K$ be a polyhedral complex such that $|K|=\mathbb{R}^{n}$. If a digital object $M$ in $K$ is a connected digital $(n-1)$-manifold without boundary, then its complement is divided into two ccomponents. Moreover, if $M$ is finite then one of the c-components is finite.

As it was mentioned above, the architecture introduced in this section allows us to translate the digital statement of the above theorem to a continuous one. Then, to prove this theorem it is enough to guarantee that the thesis of the continuous one (III.11.17 in [8] and 8.3.6 in [4]) can be translated back to the device level.

This is a general method for proving results in digital topology, but some times one fails to find in literature a continuous result which matches exactly the translated digital one. In these cases some previous work must be done to obtain such a continuous result. Our present goal is an example of this situation. So that, in Section 4 we include an alternative proof of a well-known result in topology which can be more easily translated to the device level.

From now on, unless otherwise is said, $M$ will stand for a finite connected digital (n-1)-manifold without boundary in the standard cubical decomposition $R^{n}$ of $\mathbb{R}^{n}$. In order to simplify notations, $M$, and any other digital object in $R^{n}$, will be identified with the subset of points in $\mathbb{Z}^{n}$ which are the centroids of its $n$-cubes. As an extreme case of this, a digital point, i.e. a digital object consisting of one $n$-cube $\sigma \in R^{n}$, will be identified with its centroid $c(\sigma) \in \mathbb{Z}^{n}$. 

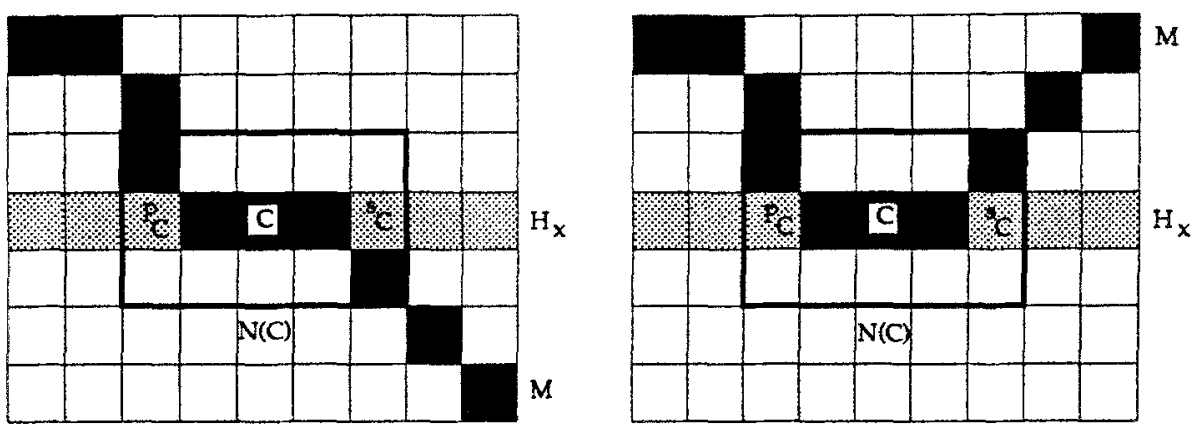

Fig. 2. A transversal intersection and a tangency.

\section{Characterizing the c-components of the complement of a digital $(\boldsymbol{n}-\mathbf{1})$-manifold in $\boldsymbol{R}^{n}$}

As in the continuous case, our characterization of the c-components of the complement of a digital manifold in $R^{n}$ is based on the notion of transversal intersection between a half-line and the manifold. Despite that in the continuous case this notion is well defined for any half-line, only for those half-lines parallel to coordinate axes it has an immediate translation to the device level. Following our outlook, what allows us to define a digital notion of transversal intersection for this type of half-lines is contained in Lemma 2 below.

In what follows, $H_{x}$ will denote the digital half-line consisting of the points $\left(x_{1}, \ldots, x_{n-1}, \lambda\right)$ in $\mathbb{Z}^{n}$ with $\lambda \geq x_{n}$, where $x=\left(x_{1}, \ldots, x_{n}\right)$ is an arbitrary point of $\mathbb{Z}^{n}$. Notice that the continuous analogue $\mathcal{H}_{x}=\left|\mathcal{A}_{H_{x}}\right|$ of $H_{x}$ is the half-line $\mathcal{H}_{x}=\left\{\left(x_{1}, \ldots, x_{n-1}, \lambda\right) \mid \lambda \in \mathbb{R}, \lambda \geq x_{n}\right\}$ in $\mathbb{R}^{n}$.

Lemma 1. (see [2]) For any digital object $O$ in a digital space $K, D$ is a connected component of the continuous analogue $\left|\mathcal{A}_{O}\right|$ if and only if $D=\left|\mathcal{A}_{C}\right|$ is the continuous analogue of a component $C$ of $O$.

Lemma 2. 1) Let $O$ and $O^{\prime}$ be two digital objects in a digital space. The continuous analogue $\left|\mathcal{A}_{O n O^{\prime}}\right|$ of the intersection $O \cap O^{\prime}$ of these objects is contained in the intersection of their continuous analogues $\left|\mathcal{A}_{O}\right| \cap\left|\mathcal{A}_{O^{\prime}}\right|$.

2) If $H_{x}$ is a digital half-line in $R^{n}$ which is parallel to one of the coordinate axes, then $\left|\mathcal{A}_{H_{x} \cap O^{\prime}}\right|=\left|\mathcal{A}_{H_{x}}\right| \cap\left|\mathcal{A}_{O^{\prime}}\right|=\mathcal{H}_{x} \cap\left|\mathcal{A}_{O^{\prime}}\right|$. In addition, the components of $H_{x} \cap O^{\prime}$ are digital points and segments.

Proof. The proof of (1) follows directly from the definition of simplicial analogue. The first part in (2) is straightforward from the fact that in $R^{n}$ every $(n-1)$-cell is face of exactly two different $n$-cells. And to prove the final part, it is enough to use that each component of the continuous analogue of a digital object is the continuous analogue of a component of that object (see Lemma 1).

We are now ready to define a function $i_{d i g}(-; M)$ from the set of digital points in the complement of a digital $(n-1)$-manifold $M$ into the set of integer numbers. 
For this we need more notation. Given an $n$-cube $\sigma \in R^{n}$ let $N(\sigma) \subseteq \mathbb{Z}^{n}$ denote the set of all points in $\mathbb{Z}^{n}$ which are the centroids of $n$-cubes of $R^{n}$ intersecting $\sigma\left(c(\sigma)\right.$ itself included). We call $N(\sigma)$ the cuboid of $\sigma$ in $\mathbb{Z}^{n}$. The cuboid of a component $C \subseteq H_{x} \cap M$ is the union $N(C)=\cup\{N(\sigma) \mid \sigma \in C\}$. Moreover, given $C$ we can consider the predecessor $p_{C}$ and the successor $s_{C}$ in $H_{x}$ of the digital points in $C$. Notice that $p_{C}$ and $s_{C}$ are both in the relative complement $N(C)-M$ of $M$ in the digital subspace induced by $N(C)$.

Definition 3. The digital index of a digital point $x \in \mathbb{Z}^{n}-M$ is $i_{d i g}(x ; M)=$ $\sum_{C} i_{d i g}(C ; M)$, where $C$ ranges over the set of connected components of $H_{x} \cap M$ and $i_{\text {dig }}(C ; M)$ is defined as follows. We write $i_{\text {dig }}(C ; M)=0$ and say that $H_{x}$ is tangent to $M$ at $C$ if its predecessor $p_{C}$ and successor $s_{C}$ are in the same $c$-component of the relative complement $N(C)-M$ of $M$ in $N(C)$. Otherwise we write $i_{d i g}(C ; M)=1$ and say that $H_{x}$ is transversal to $M$ at $C$.

The following result characterizes the points in each c-component of $\mathbb{Z}^{n}-M$. Its proof will be given in Section 5 .

Theorem 4. Let $M$ be a finite connected digital $(n-1)$-manifold without boundary in $R^{n}$. A digital point $x \in \mathbb{Z}^{n}-M$ belongs to the finite c-component of the complement of $M$ if and only if $i_{d i g}(x ; M)$ is odd.

\section{A topological criterion to determining components}

It is part of the folklore concerning the Jordan-Brouwer Theorem that the component of a point $x$ in the complement of an $(n-1)$-manifold $M \subseteq \mathbb{R}^{n}$ is characterized by the number of points in $L \cap M$, where $L$ is a half-line emanating from $x$ which intersects $M$ transversally. However, it is not easy to find in literature a general and precise proof of this fact (see, for example, chapter 9 in [3] for one of these proofs). In any case, all of these proofs make use of $\varepsilon$-movements to avoid degenerated cases. But this kind of movements can be hardly done in a digital space. So that, in this Section we will give a new proof which does not make use of any displacement of objects and, thus, can be more easily translated to digital topology.

By a (combinatorial) n-ball ( $n$-sphere, respectively) we mean a polyhedron $|K|$ such that certain subdivision of $K$ is simplicially isomorphic to a subdivision of the $n$-simplex (the boundary of the $(n+1)$-simplex, respectively). A (combinatorial) $n$-manifold is a polyhedron $|M|$ such that the link $l k(\sigma ; M)$ of each $k$-simplex $\sigma \in M$ is either a combinatorial $(n-k-1)$-ball or a combinatorial $(n-k-1)$-sphere. Here the link of $\sigma$ is the set $l k(\sigma ; M)=\{\alpha \in M \mid$ $\alpha \cap \sigma=\emptyset$ and there exists $\beta \in M$ with $\sigma<\beta, \alpha<\beta\}$. The boundary of $M$ is the subcomplex $\partial M=\{\sigma \in M \mid l k(\sigma ; M)$ is a ball $\}$.

In this section $M$ will stand for a closed (i.e. compact and without boundary) connected $(n-1)$-manifold $M \subseteq \mathbb{R}^{n}$. Then, we have the following well-known separation theorem. 
Theorem 5. (see [4, VIII.4.8]) If $M \subseteq \mathbb{R}^{n}$ is a closed ( $\left.n-1\right)$-manifold then $\mathbb{R}^{n}-M$ has two components, one bounded $B$, and one unbounded $U$. Moreover $M$ is the common boundary of both components.

For each point $x \in \mathbb{R}^{n}-M$, we denote by $\mathcal{S}_{x} \subseteq \mathbb{R}^{n}$ an arbitrary half-line starting at $x$. Then, the following lemma is easily checked.

Lemma 6. The intersection $\mathcal{S}_{x} \cap M$ consists of a union of a (possibly empty) finite family of pairwise disjoint arcs and points.

A relative ball in $\left(\mathbb{R}^{n}, M\right)$ is a pair of balls $\left(B^{n}, B^{n-1}\right)$ such that $B^{n-1} \subseteq B^{n}$, $B^{n-1} \cap \partial B^{n}=\partial B^{n-1}$ and $B^{n-1}=B^{n} \cap M$.

Lemma 7. Any relative ball $\left(B^{n}, B^{n-1}\right)$ in $\left(\mathbb{R}^{n}, M\right)$ verifies that $B^{n}-B^{n-1}$ has exactly two components each of which is contained in a distinct component of $\mathbb{R}^{n}-M$.

Proof. That $B^{n}-B^{n-1}$ has two components is an immediate consequence of a general separation theorem for manifolds with boundary proved in [10, Th. 3].

Moreover each component of $B^{n}-B^{n-1}$ is in a distinct component of $\mathbb{R}^{n}-M$. Otherwise if $B^{n}-B^{n-1} \subseteq U$, where $U$ is the unbounded component of $\mathbb{R}^{n}-M$, then $B^{n} \subseteq \bar{U}=U \cup M$ and hence there exists a point $x \in$ int $B^{n}$ with $x \in M=\operatorname{Fr}(U)$. This is a contradiction because $x$ would be an interior point of $\bar{U}$. Similarly if $B^{n}-B^{n-1} \subseteq B$, with $B$ the bounded component of $\mathbb{R}^{n}-M$.

Let $\mathcal{B}(x)=\left\{\left(B_{C}^{n}, B_{C}^{n-1}\right)\right\}$ be a family of relative balls in $\left(\mathbb{R}^{n}, M\right)$ where $C$ ranges over the set of components of $\mathcal{S}_{x} \cap M$. We say that $\mathcal{B}(x)$ is an admissible family of relative balls for $\mathcal{S}_{x} \cap M$ if $B_{C}^{n} \cap \mathcal{S}_{x}$ is an arc containing $C$ in its interior and int $B_{C}^{n} \cap$ int $B_{D}^{n}=\emptyset$ for all $C, D \subseteq \mathcal{S}_{x} \cap M$.

Lemma 8. The family $\mathcal{B}(x)$ always exists.

Proof. For each component $C \subseteq \mathcal{S}_{x} \cap M$ we can consider a regular neighbourhood $N_{C}$ of $C$ in $\mathbb{R}^{\mathfrak{n}}$ such that $N_{C}^{\prime}=N_{C} \cap M$ is a regular neighbourhood of $C$ in $M$ and $\mathcal{S}_{x} \cap N_{C}$ is a regular neighbourhood of $C$ in $\mathcal{S}_{x}$. Since $C$ is collapsible, from a well-known result from polyhedral topology (see [12, p. 41]) we get that $\left(N_{C}, N_{C}^{\prime}\right)$ is a relative ball and, moreover, $\mathcal{S}_{x} \cap N_{C}$ is an arc.

Finally we choose $N_{C}$ small enough to guarantee $N_{C} \cap N_{D}=\emptyset$ for $C \neq D$.

Now we are ready to define the (topological) index of a point in the complement of the combinatorial manifold $M$.

Definition 9. The (topological) index of a point $x \in \mathbb{R}^{n}-M$ is $i_{\text {top }}(x ; M)=$ $\sum_{C} i_{\text {top }}(C ; M)$, where $C$ ranges over the set of components of $\mathcal{S}_{x} \cap M$ and $i_{\text {top }}(C ; M)$ is defined as follows. Let $\mathcal{B}(x)=\left\{\left(B_{C}^{n}, B_{C}^{n-1}\right)\right\}$ be an admissible family of relative balls for $\mathcal{S}_{x} \cap M$. We write $i_{\text {top }}(C ; M)=0$ and say that $\mathcal{S}_{x}$ is tangent to $M$ at $C$ if the difference $B_{C}^{n} \cap \mathcal{S}_{x}-C$ (which is the union of two disjoint segments) is contained in one component of $B_{C}^{n}-B_{C}^{n-1}$. Otherwise we 
say that $\mathcal{S}_{x}$ is transversal to $M$ at $C$ and we write $i_{\text {top }}(C ; M)=1$. It can be proved, by Lemma 7 , that this definition does not depend on the choice of the family $\mathcal{B}(x)$.

Next we characterize the points in a component of $\mathbb{R}^{n}-M$ in terms of the index defined above.

Theorem 10. The point $x \in \mathbb{R}^{n}-M$ lies in the bounded component $B$ if and only if $i_{\text {top }}(x ; M)$ is odd.

Proof. Since $M$ is compact there exists a last point $x^{\prime} \in \mathcal{S}_{x} \cap M$ such that $\mathcal{S}_{x} \cap M$ is contained in the arc from $x$ to $x^{\prime}$. We order the components $C_{0}, \ldots, C_{r}$ of $\mathcal{S}_{x} \cap M$ according to the linear order on $\mathcal{S}_{x}$ determined when one identifies $t \in[0,1]$ with the point $(1-t) x^{\prime}+t x$ in $\mathcal{S}_{x}$. In particular $C_{0}$ is the component containing $x^{\prime}$.

We now choose an admissible family of relative balls $\left\{\left(B_{i}^{n}, B_{i}^{n-1}\right)\right\}_{0 \leq i \leq r}$, and let $B_{i}^{n} \cap \mathcal{S}_{x}$ the ordered segment $\left[p_{i}, q_{i}\right]$. Since the components of $B_{i}^{n}-B_{i}^{n-1}$ determine, by Lemma 7 , the components of $\mathrm{IR}^{n}-M$ then $\mathcal{S}_{x}$ locally crosses $B_{i}^{n-1}$ if and only if $p_{i}$ and $q_{i}$ are in distinct components of $\mathbb{R}^{n}-M$. As $p_{0}$ lies in the unbounded component $U$ due to the election of $x^{\prime}$ and because $p_{i+1}$ and $q_{i}, 0 \leq i \leq r-1$, are in the same component of $\mathbb{R}^{n}-M$, it follows that $q_{r} \in B$, and so $x \in B$, if and only if $i_{\text {top }}(x ; M)$ is odd.

\section{A proof of Theorem 4}

In this Section we will prove our main result. To do this, the crucial step is to find an admissible family of relative balls which is suitable for translating the continuous result (Theorem 10) to the digital one (Theorem 4). Given a digital $(n-1)$-manifold $M$ in $R^{n}$ and a digital point $x=\left(x_{1}, \ldots, x_{n}\right) \in \mathbb{Z}^{n}-M$, the required family of relative balls will be determined by the cuboids $N(C)$ of components $C$ of $H_{x} \cap M$, where $H_{x}$ is the digital half-line $\left\{\left(x_{1}, \ldots, x_{n-1}, \lambda\right) \in\right.$ $\left.\mathbb{Z}^{n} \mid \lambda \geq x_{n}\right\}$. The following result states this fact.

Proposition 11. The pairs $\left(\left|\mathcal{A}_{N(C)}\right|,\left|\mathcal{A}_{N(C) \cap M}\right|\right)$, when $C$ ranges over the set of connected components of $H_{x} \cap M$, yield an admissible family of relative balls for $\left|\mathcal{A}_{H_{x} \cap M}\right|=\left|\mathcal{A}_{H_{x}}\right| \cap\left|\mathcal{A}_{M}\right|=\mathcal{H}_{x} \cap\left|\mathcal{A}_{M}\right|$. Moreover, each c-component of the complement of $M$ meets $N(C)$ in a c-component of the relative complement $N(C)-M$ of $M$ in the digital space generated by $N(C)$.

The proof of this proposition requires some technical results which are given below. In order to simplify notations, in what follows we consider a fixed but arbitrary component $C$ of the intersection $H_{x} \cap M$, and we will denote the cuboid $N(C)$ of $C$ simply by $N$. We start with the following crucial property of the simplicial analogue $A_{N}$ of $N$.

Let $\operatorname{st}(L ; J)=\{\alpha \in J \mid$ there exists $\beta \in J$ with $\beta \cap|L| \neq \emptyset$ and $\alpha<\beta\}$ be the star of the subcomplex $L$ in the simplicial complex $J$. 
Lemma 12. A centroid $c(\mu)$ in the simplicial analogue $\mathcal{A}_{R^{n}}$ belongs to $\mathcal{A}_{N}$ if and only if all $n$-cubes containing $\mu$ are in $N$. Moreover

$$
\operatorname{st}\left(\mathcal{A}_{C} ; \mathcal{A}_{N}\right)=\operatorname{st}\left(\mathcal{A}_{C} ; \mathcal{A}_{R^{n}}\right)
$$

Proof. As it was remarked in Section 2, for any cell $\mu \in R^{n}$ its centroid $e(\mu)$ belongs to $\mathcal{A}_{R^{n}}$. Therefore if all n-cubes containing $\mu$ are in $N$ we get $c(\mu) \in \mathcal{A}_{N}$. Conversely, if $c(\mu) \in \mathcal{A}_{N}$ and there exists an $n$-cube $\sigma^{\prime} \notin N$ with $\mu<\sigma^{\prime}$, then $\mu \cap C=\emptyset$. So that, for each $n$-cube $\sigma \in N$ with $\mu<\sigma$ one can find a $(n-1)$-face $\xi<\sigma$ containing $\mu$ which is not the face of any other $n$-cube in $N$. In fact $\xi$ defines a hyperplane $H_{\xi}$ such that all $n$-cubes containing $\mu$ meet $H_{\xi}$ in a $(n-1)$ face and hence these cubes share a face of greater dimension than $\mu$. This is a contradiction, and the proof of the first part is finished.

In the second part only the inclusion $\operatorname{st}\left(\mathcal{A}_{C} ; \mathcal{A}_{R^{n}}\right) \subseteq \operatorname{st}\left(\mathcal{A}_{C} ; \mathcal{A}_{N}\right)$ needs to be checked. If $c(\mu)$ is a centroid of $\operatorname{st}\left(\mathcal{A}_{C} ; \mathcal{A}_{R^{n}}\right)-\mathcal{A}_{C}$ then there exists a 1-simplex $\langle c(\mu), c(\rho)\rangle$ where $c(\rho) \in \mathcal{A}_{C}$; that is, either $\rho$ is an $n$-cube in $C$ or $\rho$ is the common face of two $n$-cubes $\sigma_{1}, \sigma_{2} \in C$. In any case all $n$-cubes which define $\mu$ meet some cube in $C$ and hence they are cubes in $N$. From the first part we get $c(\mu) \in \mathcal{A}_{N}$.

Lemma 13. For any digital object $O$ in $R^{n}$ with $C \subseteq O$ we have $\mathcal{A}_{O n N}=$ $\mathcal{A}_{O} \cap \mathcal{A}_{N}$. Moreover

$$
\operatorname{st}\left(\mathcal{A}_{C} ; \mathcal{A}_{O \cap N}\right)=\operatorname{st}\left(\mathcal{A}_{C} ; \mathcal{A}_{N}\right) \cap \mathcal{A}_{O}=\operatorname{st}\left(\mathcal{A}_{C} ; \mathcal{A}_{O}\right)
$$

Furthermore if $T(C ; O)$ denotes $s t\left(\mathcal{A}_{C} ; \mathcal{A}_{O}\right)$ then

$$
\mathcal{A}_{O \cap N}=\operatorname{st}\left(T(C ; O) ; \mathcal{A}_{O}\right)
$$

Finally if $O$ is a digital $k$-manifold then $T(C ; O)$ is a combinatorial $k$-ball with boundary

$$
\partial T(C ; O)=\left\{\left\langle c\left(\mu_{1}\right), \ldots, c\left(\mu_{s}\right)\right\rangle \mid \mu_{s}<\sigma \cap \sigma^{\prime}, \sigma \in C, \sigma^{\prime} \notin C\right\}
$$

Proof. Clearly $\mathcal{A}_{N \text { no }} \subseteq \mathcal{A}_{O} \cap \mathcal{A}_{N}$. If $c(\mu) \in \mathcal{A}_{N}$ then any $n$-cube in $R^{n}$ containing $\mu$ belongs to $N$ by Lemma 12 . Since $c(\mu)$ also lies in $\mathcal{A}_{o}$, any $n$-cube in $O$ containing $\mu$ necessarily belongs to $N$ and so $c(\mu) \in \mathcal{A}_{O \cap N}$. The definition of simplicial analogue yields $\mathcal{A}_{O} \cap \mathcal{A}_{N} \subseteq \mathcal{A}_{O \cap N}$. The second equality of the lemma follows from the first part, Lemma 12 and the well known equality $\operatorname{st}\left(L ; J_{1} \cap J_{2}\right)=$ $\operatorname{st}\left(L ; J_{1}\right) \cap J_{2}$ when $J_{i}(i=1,2)$ are subcomplexes of $J$ containing $L$.

Now we prove (2). Let $c(\mu) \in \mathcal{A}_{O \cap N}=\mathcal{A}_{O} \cap \mathcal{A}_{N}$. Then all $n$-cubes containing $\mu$ are in $N$ by Lemma 12 . This implies that there necessarily exists an $n$-cube $\sigma_{0} \in C$ with $\mu \cap \sigma_{0} \neq \emptyset$. Also $\mu$ is the intersection of $n$-cubes $\sigma_{1}, \ldots, \sigma_{m}$ in $O$, so $r=\mu \cap \sigma_{0}=\sigma_{1} \cap \ldots \cap \sigma_{m} \cap \sigma_{0}$ and since $C \subseteq O$ it follows $c(\tau) \in \mathcal{A}_{O}$. Moreover, as $\left(c(\tau), c\left(\sigma_{0}\right)\right) \in \mathcal{A}_{O}$ we have $c(\tau) \in T(C ; O)$ and hence $\langle c(\tau), c(\mu)\rangle \in \operatorname{st}\left(T(C ; O) ; \mathcal{A}_{O}\right)$. Conversely, if $\alpha \in \operatorname{st}\left(T(C ; O) ; \mathcal{A}_{O}\right)$ is a face of $\left\langle c\left(\rho_{1}\right), \ldots, c\left(\rho_{t}\right)\right\rangle=\beta \in \mathcal{A}_{O}$ with $c\left(\rho_{i_{0}}\right) \in \beta \cap T(C ; O)$, it follows that $\rho_{1}<\rho_{i_{0}}<\sigma$ for some $\sigma \in C$. Therefore all cubes $\rho_{j}(1 \leq j \leq t)$ meet $\sigma$ and so all $n$-cubes of $O$ which define $c\left(\rho_{j}\right) \in \mathcal{A}_{O}$ are in $N$. Therefore $\alpha<\beta \in \mathcal{A}_{O \cap N}$. 
In order to prove the third part of the lemma we proceed inductively as follows. Let $t$ be the number of $n$-cubes in $C$. If $t=1$ then $C=\{\sigma\}$ and $\mathcal{A}_{C}$ is the centroid $c(\sigma)$. Then $T(C ; O)$ is the star of $c(\sigma)$ in $\mathcal{A}_{O}$; that is, a combinatorial $k$-ball. Moreover $\partial T(C ; O)=\left\{\left\langle c\left(\mu_{1}\right) \ldots c\left(\mu_{s}\right)\right\rangle \in \mathcal{A}_{o} \mid \mu_{s}<\sigma\right\}$. Assume we have already proved the result for $t-1$. If $C_{1}=C-\left\{\sigma_{t}\right\}$ then both $T\left(C_{1} ; O\right)$ and $T\left(\left\{\sigma_{t}\right\} ; O\right)$ are $k$-balls, and $T(C ; O)=T\left(C_{1} ; O\right) \cup T\left(\left\{\sigma_{t}\right\} ; O\right)$ with $T\left(C_{1} ; O\right) \cap T\left(\left\{\sigma_{t}\right\} ; O\right)=\operatorname{st}\left(c(\mu) ; \partial s t\left(c\left(\sigma_{t}\right) ; \mathcal{A}_{O}\right)\right)$ where $\mu$ is the unique $(n-1)-$ face of $\sigma_{t}$ in contact with $C_{1}$. Now $\operatorname{st}\left(c(\mu) ; s t\left(c\left(\sigma_{t}\right) ; \mathcal{A}_{O}\right)\right)$ is a combinatorial $(k-1)$-ball in the $(k-1)$-sphere $\partial s t\left(c\left(\sigma_{t}\right) ; \mathcal{A}_{O}\right)$. Hence by $[12,3.16] T(C ; O)$ is a $k$-ball. The formula of the boundary also follows inductively since $\partial T(C ; O)$ is the union

$$
\begin{gathered}
\overline{\partial T\left(C_{1} ; O\right)-s t\left(c(\mu) ; \partial s t\left(c\left(\sigma_{t}\right) ; \mathcal{A}_{O}\right)\right)} \cup \overline{\partial T\left(\left\{\sigma_{t}\right\} ; O\right)-s t\left(c(\mu) ; \partial s t\left(c\left(\sigma_{t}\right) ; \mathcal{A}_{O}\right)\right)}= \\
\left\{\left\langle c\left(\tau_{1}\right), \ldots, c\left(\tau_{s}\right)\right\rangle \in \mathcal{A}_{O} \mid \tau_{s}<\sigma \cap \sigma^{\prime}, \sigma \in C_{1}, \sigma^{\prime} \notin C_{1}, \sigma^{\prime} \neq \sigma_{t}\right\} \\
\cup\left\{\left\langle c\left(\tau_{1}\right), \ldots, c\left(\tau_{s}\right)\right\rangle \in \mathcal{A}_{O} \mid \tau_{s}<\sigma_{t}, \tau_{s} \neq \sigma_{t}, \mu\right\}= \\
\left\{\left\langle c\left(\tau_{1}\right), \ldots, c\left(\tau_{s}\right) \in \mathcal{A}_{O}\right| \tau_{s}<\sigma \cap \sigma^{\prime}, \sigma \in C, \sigma^{\prime} \notin C\right\}
\end{gathered}
$$

where $\overline{K-L}$ stands for the set of simplexes which are face of some simplex $\sigma$ in the complex $K$ with $\sigma \notin L$.

Note 14. If $O=N$ then $|T(C ; N)|=\cup\{\sigma \mid \sigma \in C\}$. Indeed, one easily checks $|T(C ; N)| \subseteq \cup\{\sigma \mid \sigma \in C\}$. Moreover, if $x \in \eta$ with $\eta$ a cube in $C$ then $x$ lies in a simplex $\langle c(\eta), \ldots, c(\sigma)\rangle \in \mathcal{A}_{R^{n}}$. Therefore $x \in \operatorname{st}\left(c(\sigma) ; \mathcal{A}_{R^{n}}\right) \subseteq|T(C ; N)|$.

In order to show that $\mathcal{A}_{O \cap N}$ is a combinatorial ball we set the following definition and lemmas.

Definition 15. Given an arbitrary digital object $O$ in $R^{n}$ we say that the cube $\tau$ is minimal with respect to $O$ if $c(\tau)$ is a minimal vertex in the conceptual level $\mathcal{C}_{O}$. Here we use the digraph structure of $\mathcal{C}_{O}$. That is, for any other cube $\mu$ either $\tau \cap \mu=\emptyset$ or $\tau<\mu$.

Lermma 16. Two minimal cubes with respect to $O$ cannot span an edge in $\mathcal{A}_{O}$.

Let us assume again that $O$ is a digital $k$-manifold in $R^{n}$ with $C \subseteq O$.

Lemma 17. If $n \geq 2$, all the centroids of minimal cubes with respect to $O \cap N$ are in $\partial T(C ; O)$.

In any case, let $\tau_{1}, \ldots, \tau_{m}$ be the set of minimal cubes with respect to $O \cap N$ which lie in $\partial T(C ; O)$. We inductively define $M_{0}^{O}=T(C ; O)$ and $M_{i}^{O}=M_{i-1}^{O} \cup$ $\operatorname{st}\left(c\left(\tau_{i}\right) ; \overline{\mathcal{A}_{O}-M_{i-1}^{O}}\right)$. Then, the following result is proved.

Lemma 18. The following properties hold

1) $c\left(\tau_{i}\right) \in \partial M_{i-1}^{O}$.

2) $M_{i}^{O} \cap \operatorname{st}\left(c\left(\tau_{i}\right) ; \overline{\mathcal{A}_{O}-M_{i-1}^{O}}\right)=\operatorname{st}\left(c\left(\tau_{i}\right) ; \partial M_{i-1}^{O}\right)$.

3) Each $M_{i}^{O}$ is a combinatorial $k$-ball.

b) $M_{m}^{O}=\mathcal{A}_{O \cap N}$.

5) $\partial \mathcal{A}_{O \cap N}=\left\{\left\langle c\left(\rho_{1}\right), \ldots, c\left(\rho_{s}\right)\right\rangle \in \mathcal{A}_{O \cap N} \mid \rho_{i} \nless \sigma\right.$ for all $\sigma \in C$ and $\left.i \leq s\right\}$. 
Proof. (1) is immediate from Lemma 16. Both (2) and (3) follow from the same inductive argument. Namely, $M_{0}^{O}$ is a $k$-ball by Lemma 13 . Assume we have proved (2) and (3) for $i-1$. Then $s t\left(c\left(\tau_{i}\right) ; \partial M_{i-1}^{O}\right)$ is a $(k-1)$-ball and $\overline{\mathcal{A}_{O}-M_{i-1}^{O}}$ is a combinatorial $k$-manifold by [12,3.14]. Now (2) is a well known formula in polyhedral topology and hence $M_{i}^{O}$ is the union of two $k$-balls which meet in a $(k-1)$-ball; and thus it is a combinatorial $k$-ball by $[12,3.16]$.

(4) Assume $n \geq 2$. The case $n=1$ is obvious. If $c(\mu) \in \mathcal{A}_{O \cap N}$ verifies $\mu<\sigma$ for some $\sigma \in C$ then $c(\mu) \in T(C ; O)=M_{0}^{O}$. If $\mu \nless \sigma$ for all $\sigma \in$ $C$, let $\alpha \in \mathcal{A}_{O}$ be a $k$-simplex with $c(\mu)$ a vertex of $\alpha$. The minimal vertex $c(\tau)$ of $\alpha$ belongs to $\partial T(C ; O)$ by Lemma 17 and hence $c(\mu) \in M_{i}^{O}$ for some $i$. Therefore $\mathcal{A}_{O \cap N} \subseteq M_{m}^{O}$. Conversely, by definition and Lemma $13(2) M_{i}^{O} \subseteq$ $\operatorname{st}\left(T(C ; O) ; \mathcal{A}_{O}\right)=\mathcal{A}_{O \cap N}$ for all $i \leq m$.

(5) Let $S C(C ; O)$ denotes the simplicial complement of $T(C ; O)$; that is

$$
\begin{aligned}
S C(C ; O) & =\left\{\beta=\left\langle c\left(\rho_{1}\right), \ldots, c\left(\rho_{t}\right)\right\rangle \in \mathcal{A}_{O} \mid \beta \cap T(C ; O)=\emptyset\right\} \\
& =\left\{\left\langle c\left(\rho_{1}\right), \ldots, c\left(\rho_{t}\right)\right\rangle \in \mathcal{A}_{O} \mid \rho_{i} \nless \sigma \text { for all } \sigma \in C\right\} .
\end{aligned}
$$

We have $S C(C ; O) \cap \mathcal{A}_{O \cap N}=\left\{\left\langle c\left(\rho_{1}\right), \ldots, c\left(\rho_{s}\right)\right\rangle \in \mathcal{A}_{O \cap N} \mid \rho_{i} \nless \sigma\right.$ for all $\sigma \in$ $C$ \}. By using Lemma $13(2)$ it is easy to check that the complex $S C(C ; O)$ triangulates the topological closure $\overline{\mathcal{A}_{O}-\mathcal{A}_{O \cap N}}$. In addition, since $\mathcal{A}_{O \cap N}$ and $\mathcal{A}_{O}$ are both combinatorial $k$-manifolds, it follows by $[12,3.14]$ that $S C(C ; O)$ is a combinatorial $k$-manifold with boundary $\partial S C(C ; O)=\partial \mathcal{A}_{O \cap N}=S C(C ; O) \cap \mathcal{A}_{O \cap N}$.

Proof of Proposition 11. The first part of Proposition 11 is now immediate since $\mathcal{A}_{N(C)}$ and $\mathcal{A}_{N(C) \cap M}$ are balls with $\partial \mathcal{A}_{N(C) \cap M}=\partial \mathcal{A}_{N(C)} \cap \mathcal{A}_{M}$ by Lemma 18(4) and (5). The second part follows from Lemma 7 and the fact that each $c$-component of the complement of a digital object $O$ consists of the $n$-cells whose centroids are in the complement of its continuous analogue $\left|\mathcal{A}_{O}\right|$.

Now we are ready to prove our main result by relating the digital index $i_{\text {dig }}(x ; M)$ of a digital point $x$ in the complement $\mathbb{Z}^{n}-M$ of a digital $(n-1)$ manifold $M$ to its topological index $i_{\text {top }}\left(x ;\left|\mathcal{A}_{M}\right|\right)$ as a point in the complement $\mathbb{R}^{n}-\left|\mathcal{A}_{M}\right|$ of the combinatorial manifold $\left|\mathcal{A}_{M}\right|$.

Lemma 19. (see [2]) Let $M$ be a finite connected digital (n-1)-manifold without boundary in $R^{n}$. A digital point $x$ belongs to the finite c-component of the complement $\mathbb{Z}^{n}-M$ of $M$ if and only if $x$ belongs to the bounded component of $\mathbb{R}^{n}-\left|\mathcal{A}_{M}\right|$.

Proof of Theorem 4. According to Theorem 10 and Lemma 19 it will suffice to show the identity $i_{\text {dig }}(x ; M)=i_{\text {top }}\left(x ;\left|\mathcal{A}_{M}\right|\right)$ since the $c$-components of the complement $\mathbb{Z}^{n}-M$ of $M$ are determined by the components of $\mathbb{R}^{n}-\left|\mathcal{A}_{M}\right|$. Here we use Lemmas 2 and 1 to check that $C$ is a component of the intersection $H_{x} \cap M$ if and only if $\left|\mathcal{A}_{C}\right|$ is a component of $\left|\mathcal{A}_{H_{x} \cap M}\right|=\left|\mathcal{A}_{H_{x}}\right| \cap\left|\mathcal{A}_{M}\right|=\mathcal{H}_{x} \cap\left|\mathcal{A}_{M}\right|$. So that, the previous identity is obtained by proving $i_{\text {dig }}(C ; M)=i_{\text {top }}\left(C ;\left|\mathcal{A}_{M}\right|\right)$ for each component $C$ of $H_{x} \cap M$. Now we use the admissible family of relative balls $\left\{\left(\left|\mathcal{A}_{N(C)}\right|,\left|\mathcal{A}_{N(C) \cap M}\right|\right)\right\}$ for $\mathcal{H}_{x} \cap\left|\mathcal{A}_{M}\right|$, given in Proposition 11, and 
observe that the predecessor $p_{C}$ and successor $s_{C}$ of $C$ are each one of them contained in a different segment of $\left|\mathcal{A}_{N(C)}\right| \cap \mathcal{H}_{x}-\left|\mathcal{A}_{C}\right|$. And finally, as the $c$-components of the relative complement $N(C)-M=N(C)-N(C) \cap M$ of $M$ in the digital space generated by $N(C)$ are determined by the components of $\left|\mathcal{A}_{N(C)}\right|-\left|\mathcal{A}_{N(C) \cap M}\right|, p_{C}$ and $s_{C}$ are in the same $c$-component of $N(C)-M$ if and only if the two segments in $\left|\mathcal{A}_{N(C)}\right| \cap \mathcal{H}_{x}-\left|\mathcal{A}_{C}\right|$ are in the same component of $\left|\mathcal{A}_{N(C)}\right|-\left|\mathcal{A}_{N(C) \cap M}\right|$.

\section{A simplified algorithm for the 3-dimensional case.}

In [1] it is proved that 2-manifolds in $R^{3}$ and $(26,6)$-surfaces in the sense of Morgenthaler-Rosenfeld [9] are the same. From this, we will observe that our method to determining the $c$-components of the complement of a digital $(n-1)$ manifold $M$ in $R^{n}$ coincides, for the case $n=3$, with that method given in [9] by Morgenthaler-Rosenfeld to determining the 6-components of the complement of a $(26,6)$-surface.

Firstly, notice that our definition of transversal intersection essentially agrees, up to the notions of connection used, with the one given in [9]. Because of this, it will suffice to show that these notions coincide not only in the ambient space $R^{3}$ but also in the digital subspaces generated by $N(C)$, where $C$ is a connected component of $H_{x} \cap M$.

From definitions in Section 2 it is immediate that our notion of connection in $R^{n}$, and its subspaces, agrees with the notion of $\left(3^{n}-1\right)$-adjacency in $\mathbb{Z}^{n}$. In fact, following this language, a component of a digital object $O \subseteq R^{n}$ is just a $\left(3^{n}-1\right)$-component of $O$, when $O$ is regarded as a subset of $\mathbb{Z}^{n}$. On the other hand, in [2] it is proved that a c-component of the complement of $O$ in $R^{n}$ is a $2 n$-component of $\mathbb{Z}^{n}-O$; and the proof can be easily extended to show that these notions also coincide for complements of objects in the digital subspace generated by $N(C)$.

In this way, Theorem 4 shows that a point $x$ in the complement $\mathbb{Z}^{n}-M$ of a finite $\left(3^{n}-1,2 n\right)$-hypersurface $M$ in $\mathbb{Z}^{n}$ belongs to the finite $2 n$-component of $\mathbb{Z}^{n}-M$ if and only if $i_{\text {dig }}(x ; M)$ is odd.

Finally, we are going to introduce an important simplification for the case $n=$ 3 . In the general case, the index of a digital point $x \in \mathbb{Z}^{n}-M$ can be computed in $O\left(3^{n-1} K+L\right)$ time, where $K$ is the number of points in the intersection $H_{x} \cap M$ and $L$ is a constant which depends on the size of the digital picture representing $M$. The algorithm scans along the line $H_{x}$ to get the components $C$ of $H_{x} \cap M$, and then computes the $c$-components of $N(C)-M$ by using depth-first search in the graph of $2 n$-adjacencies with vertex set $N(C) \subseteq \mathbb{Z}^{n}$. On the other hand, Theorem 20 below shows that to compute the index of a digital point $x \in \mathbb{Z}^{3}-M$ it is enough to study certain planes containing the predecessor $p_{C}$ and successor $s_{C}$ of each component $C$ of $H_{x} \cap M$, which can be done in $O(t+L)$ time where $t$ is the number of such components. So that, to compute the $c$-components of $N(C)-M$ is no longer needed for the case $n=3$. 


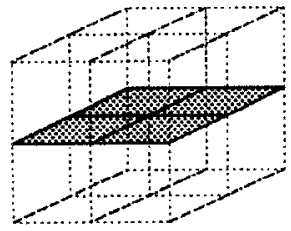

(a)

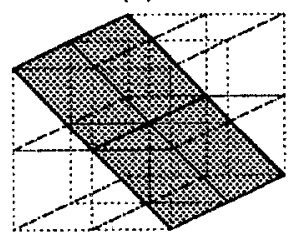

(e)

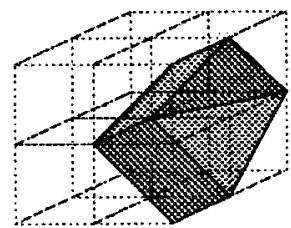

(i)

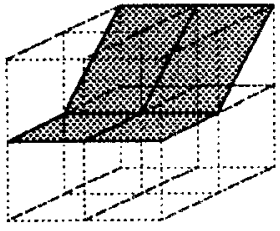

(b)

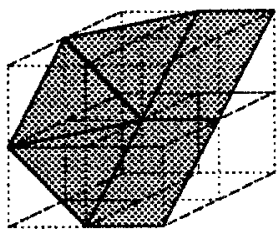

(f)

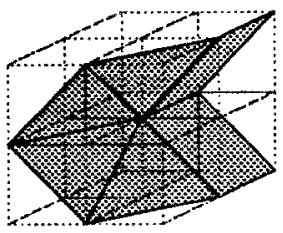

(j)

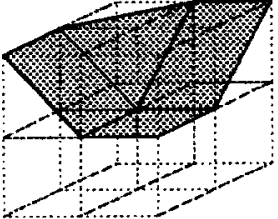

(c)

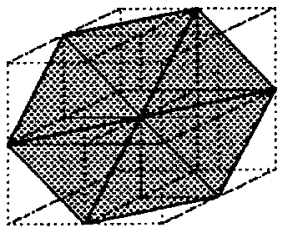

(g)

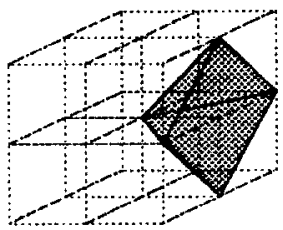

(k)

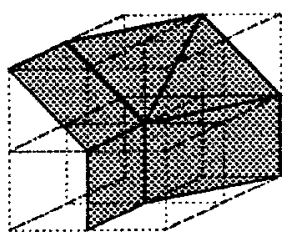

(d)

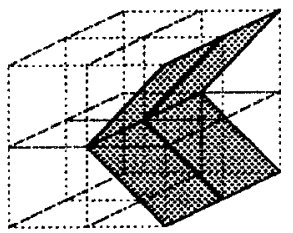

(h)

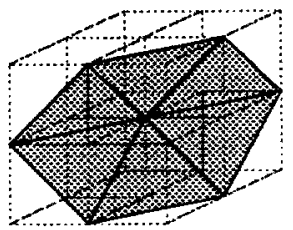

(l)

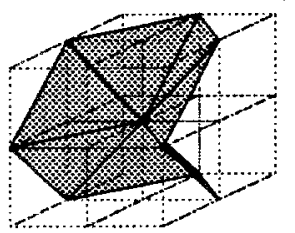

(m)

Fig. 3. Cycle plates at a point of a $(26,6)$-surface.

This simplification is based on the characterization of $(26,6)$-surfaces in terms of cycle plates due to Kong-Roscoe [7].

Let $C$ be a component of $H_{x} \cap M$, and let $P_{C}, S_{C} \subseteq \mathbb{Z}^{3}$ the orthogonal planes to $H_{x}$ which contain the predecessor $p_{C}$ and the successor $s_{C}$ of $C$, respectively.

Theorem 20. The digital half-line $H_{x}$ is tangent to $M$ at $C$ (i.e.: $i_{\text {dig }}(C ; M)=$ $0)$ if and only if the intersection $P_{C} \cap M \cap N(C)$ is non-empty and $S_{C} \cap M \cap N(C)$ is the image of $P_{C} \cap M \cap N(C)$ by the obvious translation along $H_{x}$.

Proof. Figure 3 shows all possible (up to rotations or symmetries) cycle plates at a digital point $x$ of a $(26,6)$-surface. So, if $C$ is a digital point then $H_{x}$ is tangent to $M$ at $C$ if and only if the cycle plate at $C$ is either one from (h)-(m) in Figure 3 or any other obtained from it by a rotation or symmetry which preserves the property established in Theorem 20.

In case $C$ is a digital segment one checks that for all planes $P$ between $P_{C}$ and $S_{C}$ the intersections $P \cap M \cap N(C)$ are the images of each other by a translation along $H_{x}$ (see Figure 4 for some examples). Therefore the tangency depends only 

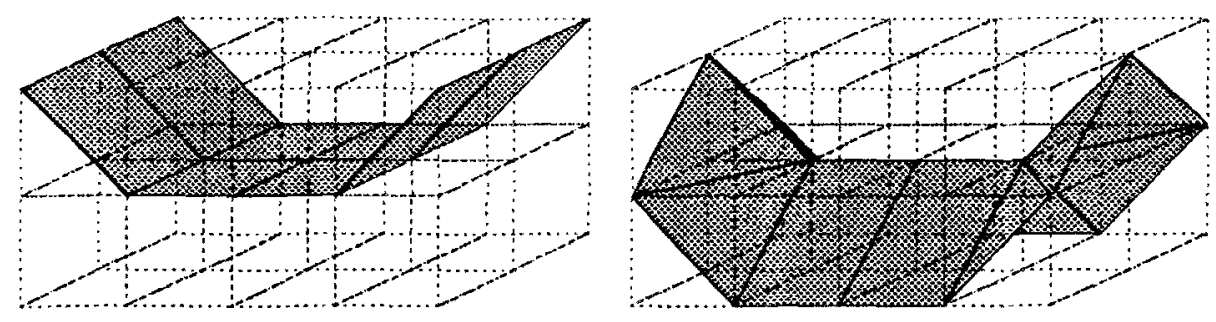

Fig. 4.

on the intersections $P_{C} \cap M \cap N(C)$ and $S_{C} \cap M \cap N(C)$ and one concludes as in the case when $C$ is a point by an exhaustive analysis of cycle plates.

\section{Acknowledgements:}

The authors are grateful to Prof. Jean Françon who posed to them the problem which has originated this paper.

This work was partially supported by the project DGICYT PB92-0672.

\section{References}

1. R. Ayala, E. Domínguez, A.R. Francés, A.Quintero, J. Rubio. On surfaces in digital topology. Proceedings of the 5 th Colloquium on Discrete Geometry for Computer Imagery DGCI'5. 271-276 (1995).

2. R. Ayala, E. Domínguez, A.R. Francés, A.Quintero, J. Rubio. A polyhedral approach to $n$-dimensional digital topology. Preprint.

3. R.H. Bing. The geometric topology of 3-manifolds. Coll. Pub., 40. Amer. Math. Soc. 1983.

4. A. Dold. Algebraic Topology. Die Grundlehren der Math., 200. Springer, 1972.

5. E. Dominguez, A.R. Francés, A. Márquez. A Framework for Digital Topology. Proceedings of the IEEE International Conference on Systems, Man, and Cybernetics, vol. 2, 65-70, 1993.

6. E. Khalimsky, R. Kopperman, P.R. Meyer. Computer Graphics and connected topologies on finite ordered sets. Topology and its Applications, 36 (1990), 1-17.

7. T.Y. Kong, A.W. Roscoe. Continuous Analogs of Axiomatized Digital Surfaces. Computer Vision, Graphics, and Image Processing 29, 60-86 (1985).

8. W.S. Massey. Homology and Cohomology Theory. Marcel Dekker, 1978.

9. D.G. Morgenthaler, A. Rosenfeld. Surfaces in Three-Dimensional Digital Images. Information and Control 51, 227-247 (1981).

10. F. Raymond. Separation and union theorems for generalized manifolds with boundary. Michigan Math. J.7(1970) 1-21.

11. A. Rosenfeld. Digital Topology. Amer. Math. Monthly, 86 (1979), 621-630.

12. C.P. Rourke, B.J. Sanderson. Introduction to Piecewise linear topology. Ergebnisse der Math., 69. Springer, 1972. 